\title{
BMJ Open Effect of a tailored multidimensional intervention on the care burden among family caregivers of stroke survivors: a randomised controlled trial
}

\author{
Mahmoud Ahmed Elsheikh (D) ,, ${ }^{1,2}$ Michiko Moriyama, ${ }^{1}$ Md Moshiur Rahman (D) , ${ }^{1}$ \\ Mayumi Kako, ${ }^{1}$ Ahmed Hashem EL-Monshed (D) , ${ }^{3,4}$ Mohamed Zoromba, ${ }^{3}$ \\ Hamada Zehry, ${ }^{5}$ Maha Hazem Khalil, ${ }^{6}$ Abdel-Hady El-Gilany, ${ }^{7}$ Mostafa Amr ${ }^{8}$
}

To cite: Elsheikh MA, Moriyama M, Rahman MM, et al. Effect of a tailored multidimensional intervention on the care burden among family caregivers of stroke survivors: a randomised controlled trial. BMJ Open 2022;12:e049741. doi:10.1136/ bmjopen-2021-049741

- Prepublication history and additional supplemental material for this paper are available online. To view these files, please visit the journal online (http://dx.doi.org/10.1136/ bmjopen-2021-049741).

Received 01 February 2021 Accepted 06 January 2022

Check for updates

(C) Author(s) (or their employer(s)) 2022. Re-use permitted under CC BY-NC. No commercial re-use. See rights and permissions. Published by BMJ.

For numbered affiliations see end of article.

Correspondence to Dr Mahmoud Ahmed Elsheikh; d194332@hiroshima-u.ac.jp

\section{ABSTRACT}

Objectives To evaluate the effectiveness of a tailored multidimensional intervention in reducing the care burden of family caregivers of stroke survivors. This intervention considered caregivers' perceived needs and incorporated three evidence-based dimensions (psychoeducation, skillbuilding and peer support).

Design A prospective randomised control trial.

Setting A community-based study conducted in Egypt. Participants A total of 110 caregivers aged $\geq 18$ years who cared for a survivor within 6 months of stroke, with modified Rankin Scale scores of 3-5, and without other physical disabilities or terminal illnesses were recruited between December 2019 and May 2020. Participants were assigned to the intervention group (IG; $n=55)$ and control group (CG; $n=55$ ) through open-label, parallel 1:1 randomisation.

Intervention The IG was provided with tailored multidimensional interventions for 6 months until November 2020, including three home visits, six homebased telephone calls and one peer-support session. The CG received simple educational instructions at a single visit.

Outcome The participants completed the Zarit Burden Interview (primary outcome) and the WHO Quality of LifeBREF (secondary outcome) before the intervention (T0), at 3 months (T1) and at 6 months (T2).

Results No differences were observed between the characteristics of the groups at baseline (TO). The independent t-test showed no significant differences in the care burden and Quality of Life (QoL) at T1 and T2 between the groups. The intervention had no significant effect on the outcomes between or within groups over time, as shown by the repeated-measures analysis of variance. However, the group and time interaction had significant main effects on caregivers' QoL (psychological and social domains).

Conclusion The main results showed that participants in the IG did not experience an improvement in the main outcomes. Nevertheless, the improvement in the psychological and social domains may have been attributed to our intervention.

Trial registration number NCT04211662.
Strengths and limitations of this study

- The study's conceptual framework was developed based on evidence-based guidelines and scientific recommendations.

- This is the first randomised control trial that offered a tailored multidimensional intervention for the family caregivers of stroke survivors in Egypt and was designed by an interdisciplinary team.

- The extent of the intervention was inconsistent throughout the intervention period.

- Caregivers' rates of compliance with the intervention were not measured. Hence, the intervention effects could not be explored further.

- Unblinded outcome assessments and self-reported questionnaires are sources of possible bias.

\section{INTRODUCTION}

Stroke being one of the most common causes of severe disability, ${ }^{1}$ often requires long-term care. ${ }^{2}$ Stroke survivors with functional impairment depend on caregivers, usually family members, to assist them in performing their daily activities ${ }^{3}$ and other stroke management and rehabilitation tasks. ${ }^{24}$ The high level of stress experienced by family caregivers often causes them strain and burden. ${ }^{56}$ Literature reveals that family caregivers of stroke survivors often feel insufficiently supported to cope with the care burden. ${ }^{78}$ This urgent issue indicates the need for evidence-based initiatives that reduce the care burden among family caregivers of stroke survivors, ${ }^{910}$ and in turn, improving the well-being of stroke survivors as well. ${ }^{11}$

As discussed in the published protocol for the current study, ${ }^{12}$ multiple systematic reviews have investigated interventions designed to support family caregivers of stroke survivors by reducing their care burden and its consequences. ${ }^{13-15}$ However, many 
of these studies have reported mixed results. ${ }^{13-15}$ The controversies about the effectiveness of previous interventions have been attributed to several factors, particularly related to the type of the interventions. ${ }^{13}{ }^{14}$ First, most of the interventions assessed were standardised; in other words, they approached the subject with an assumption that all caregivers have the same needs. ${ }^{16}{ }^{17}$ Whereas, evidence suggests that tailored interventions-customised for the caregiver's needs-are the most feasible and have the most positive impact on caregivers and stroke survivors. ${ }^{18} 19$ The need for tailored interventions to mitigate the unmet needs of family caregivers of stroke survivors have been enthusiastically recommended in the literature. $^{2021}$

Second, regarding the components of the interventions, researchers concluded that single-approach interventions, which provide only one component of support, might have limited benefit for the family caregivers of stroke survivors. ${ }^{14}{ }^{15}$ In contrast, interventions that incorporate more than one approach, such as skillbuilding, ${ }^{4}$ psychoeducation ${ }^{10}$ and peer support, ${ }^{522}$ were likely to have the most significant effects on care burden and other adverse effects. ${ }^{13} 1423$ Therefore, empirical findings endorse conducting future studies that focus on integrating various approaches. Nonetheless, such interventions have not been tested in various settings, and little is known about their efficacy. ${ }^{513}$ Reviewing the abovementioned factors, we developed a tailored and multidimensional intervention based on an evidencebased conceptual framework that considers the previous scientific recommendations. The aim of this study was to evaluate the effectiveness of the proposed intervention in reducing the care burden of family caregivers. The study findings will add to what is known in nursing and medical practices about providing interventions to caregivers.

Tailored multidimensional intervention

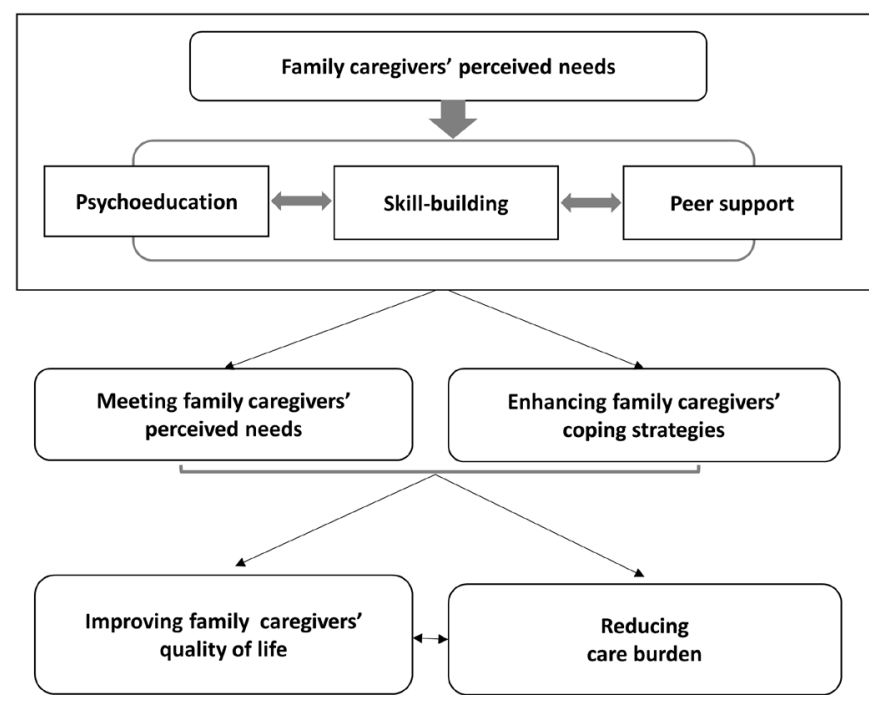

Figure 1 Conceptual framework.

\section{Conceptual framework}

Figure 1 illustrates the conceptual framework of the study. Family caregivers' perceived needs, which were considered the most typical determinants and predictors of the care burden ${ }^{24}$ and quality of life $(\mathrm{QoL})^{26}$ were the foundational elements of the proposed conceptual framework. We maintain that meeting the family caregivers' perceived needs would reduce the severity of the care burden and improve QoL. The three intervention dimensions of psychoeducation, ${ }^{27}$ skill-building ${ }^{28}$ and peer support ${ }^{29}$ were recognised as interrelated concepts. Together, the three dimensions are aimed to meet family caregivers' unmet needs, thus reducing their care burden and improving their QoL. Nevertheless, each dimension has its own strategies, as explained in the study protocol. ${ }^{12}$ For instance, the aim of psychoeducation is to reduce caregiving-related stress by providing health information according to the caregivers' unmet needs, supporting them emotionally and psychologically, and assisting them in controlling their appraisal of the current situation. Thus, we hypothesised that psychoeducation would enhance adaptive coping, ${ }^{30}$ by incorporating effective coping skills, ${ }^{31}$ and consequently reducing the care burden and improving the caregivers' perceived QoL. ${ }^{32}$

Similarly, the building of care-related skills among caregivers ${ }^{33} 34$ has been associated with reductions in the strain of the caring role ${ }^{3536}$ and improvements in QoL. ${ }^{37}$ Skillbuilding aims to enhance the caregivers' abilities and involve them actively in the stroke survivors' care through several strategies, including home visit nursing for handson-training on direct care skills, teaching caregivers about managing stroke survivors' emotions and behaviours. Moreover, skill-training also includes educating caregivers about managing their own emotions, and training them to communicate with healthcare providers and others effectively. Furthermore, skill-building motivates caregivers to seek family support, which is crucial for caregivers of patients with chronic disabilities ${ }^{38-40}$ for relieving personal strain and role strain ${ }^{4142}$ and improving QoL. ${ }^{43}$

Peer-support groups play a significant role in experiencebased learning. ${ }^{44}$ It allows peers to exchange effective methods for buffering the impacts of stressful life events and managing their changed lives. ${ }^{35} 45$ We proposed that peer support would provide caregivers with alternatives to meet their needs and develop healthy coping strategies, thereby relieving their care burden and improving QoL. ${ }^{46}$

\section{Aim and hypothesis}

This study aimed to evaluate the effectiveness of a tailored multidimensional intervention in reducing the care burden among family caregivers of stroke survivors. The main hypothesis was that the family caregivers of stroke survivors (intervention group (IG)) who received the tailored multidimensional intervention would experience a reduction in their care burden, relative to those who received an educational booklet (control group (CG)) . 


\section{METHODS AND ANALYSIS}

\section{Trial design and study participants}

A prospective, open-label, parallel 1:1 randomised controlled trial (RCT), unblinded for outcome evaluation, structured on the basis of the Consolidated Standards of Reporting Trials (CONSORT) ${ }^{47}$ was conducted in Dakahlia Governorate, Egypt ${ }^{48}$ from December 2019 to November 2020. This study aimed to report care burden as the primary outcome and QoL as a secondary outcome. Results related to other secondary outcomes will be reported in future studies.

The protocol of this study was developed in accordance with the Standard Protocol Items Recommendations for Interventional Trials. ${ }^{49}$ This has been documented in detail elsewhere, to ensure reproducibility. ${ }^{12}$ In summary, the target group of our study was family caregivers of stroke survivors. The inclusion criteria were: family caregivers who were $\geq 18$ years of age; agreed to participate in the study; caring for stroke survivors who had a stroke within the previous 6 months $s^{50}$ and needed assistance with the modified Rankin Scale (mRS) scores of 3-5 at enrolment. ${ }^{52}$ Caregivers were excluded if they had cognitive impairment or if their stroke survivors had other physical disabilities or terminal-stage illnesses.

\section{Patient and public involvement}

Patients and/or the public were not involved in the design, or conduct, or reporting, or dissemination plans of this research.

\section{Recruitment procedure and assessment of eligibility}

In this community-based study, the participants were approached by physicians and nurses at seven outpatient clinics located in Mansoura City, the capital of Dakahlia Governorate, and surrounding cities within a $30 \mathrm{~km}$ radius. The participants were recruited using the detailed steps provided in the protocol of this study. The researchers contacted caregivers via telephone to explain the purpose of the study and schedule home visits. Each home visit was conducted by one of the researchers to verify whether the selected participants met the eligibility criteria. During these visits, the researcher calculated the stroke survivor's mRS score to determine their dependence level ${ }^{52}{ }^{53}$ and measured their degree of cognitive impairment using the Mini-Mental State Examination (MMSE). ${ }^{53}$ All eligibility criteria were verified through interviews with the caregivers, assessments of the stroke survivors' health conditions, and reviews of the available medical records.

\section{Randomisation and allocation}

After confirming the eligibility criteria, the family caregivers were allocated to either the IG or the CG through 1:1 open-label randomisation. The caregivers were randomised into one of the two groups after stratifying stroke survivors according to dependency level (mRS: 3, 4 or 5$)^{5556}$ and degree of cognitive impairment (MMSE $\leq 20$ or $>20) .{ }^{53} 54$ Randomisation was conducted using a computer-generated series of numbers and performed by a member of the research group—not a part of the intervention, who then informed the researchers which participants had been assigned to which group.

\section{IG: tailored multidimensional intervention}

Each family caregiver in the IG received the proposed intervention, developed using the evidence-based conceptual framework of this study. To ensure validity, ${ }^{57}$ a tailored intervention was designed for each caregiver in response to their perceived unmet needs through the five steps described in the study protocol. ${ }^{12}$ The interventions were created by an interdisciplinary team of medical and nursing experts. ${ }^{58}$

\section{Intervention delivery and control}

The intervention was implemented taking into account evidence indicating that multiple methods of delivery might result in better outcomes ${ }^{57}$ : the intervention was delivered over 6 months (previously determined appropriate duration), ${ }^{13}$ through three $120 \mathrm{~min}$ home visits, six $40 \mathrm{~min}$ telephone calls and one $90 \mathrm{~min}$ peer-support session. ${ }^{13}{ }^{23}$ The interventions were administered by 10 intervention nurses, each with a bachelor's degree in nursing and experience working in stroke care units. The nurses all underwent a 31- hour training programme before the start of the study. Throughout the 6 months of intervention, the interdisciplinary team performed monthly checks of the nurses' documentation of the intervention progress and provided constructive feedback. An example of the nurses' documentation record format is presented as online supplemental file 1 .

The previously published study protocol extensively describes the intervention delivery. ${ }^{12}$ Moreover, as an extension of CONSORT item five ${ }^{47}$ and means of ensuring adequate reporting of the study intervention, the completed Template for Intervention Description and Replication checklist is also available (online supplemental file 2). ${ }^{59}$

\section{Control group}

An instructional booklet with information on stroke and caring for stroke survivors was delivered to the CG participants. The intervention nurses explained this information to each of the CG participants during a special home visit (separate from the visits of baseline and outcome assessments).

\section{Data collection and outcomes}

After allocating the study participants to either the IG or CG, the intervention nurses conducted an initial home visit for each group to collect data about the sociodemographic characteristics and health conditions of stroke survivors and their family caregivers. Also, the Zarit Burden Interview (ZBI) ${ }^{60}$ and WHO Quality of LifeBREF (WHOQOL-BREF) ${ }^{61}$ were administered to the family caregivers to assess the baseline care burden and QoL at T0 before the intervention. At T1 (3 months) and T2 (6 months), the nurses again collected ZBI and 
WHOQOL-BREF data, through home visits for all IG and CG participants. The outcomes were measured using selfadministered questionnaires with the assistance of nurses (if needed).

\section{Outcome measures}

Short version of ZBI

The care burden was assessed using the short version of the ZBI (12 items) which was established by Bédard $e t$ $a l,{ }^{60}$ and used to measure the family caregiver's burden of caring for stroke survivors. ${ }^{62-64}$ It was adapted and validated for Arabic language and culture (Cronbach's alpha $=0.77),{ }^{65}$ The tool consists of 12 questions, with each item rated from 0 to 4 ( $0=$ never, $1=$ rarely, $2=$ sometimes, $3=$ quite frequently and $4=$ nearly always). To calculate the care burden, the overall score was obtained from the arithmetic mean of the values equivalent to the responses to the 12 questions, while the individual score was obtained from the arithmetic mean of the values equivalent to the answers to particular questions. No cut-off point was assigned for classifying the care burden; higher score indicated higher care burden. ${ }^{60}$ Our analysis yielded a Cronbach's alpha of 0.800 .

\section{WHO Quality of Life-BREF}

The WHOQOL-BREF has been used to assess the QoL of family caregivers. ${ }^{56} 6266$ It is a 26 -item, self-rated questionnaire, involving two general questions and 24 questions covering the physical, psychological, social relationships and environmental domains. The items are rated on a 5-point Likert scale (from 1 to 5). All four domains of the instrument have been adapted and validated for Arabic language and culture (Cronbach's alpha $\geq 0.75) .{ }^{67}$ Higher scores indicate better QoL. The mean score of the items within each domain was used to calculate the domain score. Compared with the scoring of the WHOQOL-100, the mean scores were then multiplied by four to obtain the domain scores, which ranged from 0 to $100 .^{61}$ The Cronbach's alpha values for the four domains were as follows: physical, 0.864; psychological, 0.895; social relationships, 0.793 and environment, 0.794 . The overall Cronbach's alpha was 0.921 .

\section{Sample size}

The sample size was estimated using G power software V.3.1.9.4 (Psychonomic Society, Madison, Wisconsin, USA ${ }^{68}$ with an effect size of 0.72 , which was based on the results of a similar previous study that demonstrated a difference in care burden among family caregivers using ZBI. ${ }^{69}$ Assuming that the power analysis showed the difference between the two independent means (two groups), a confidence level of 0.95 , a statistical power of 0.90 , and fair division, the sample size required was 84 caregivers ( $\mathrm{n}=42$ per group). An additional 26 caregivers were recruited to account for an estimated dropout rate of $30 \% .^{7071}$ The final sample size was 110 participants (55 in the IG and 55 in the CG).

\section{Statistical analyses}

This study used an intention-to-treat analysis. ${ }^{72}$ Statistical Package for Social Sciences (SPSS) V.22.0 (IBM) was used to perform all statistical analyses. Continuous data were expressed as means and SD or as median, minimum and maximum values. Categorical data were expressed as frequencies and percentages. The baseline characteristics of the two groups were compared using the t-test or MannWhitney U test, Pearson's $\chi^{2}$ test or Fisher's exact test, according to the variable type and distribution of data. The intervention efficacy was measured by comparing the means of the outcomes (ZBI and WHOQOL-BERF) between the two groups at separate time points: T0, T1, and T2; independent t-tests were conducted for the same. In addition, to assess changes in outcomes within the groups over time (T0-T2), a two-way repeated measures analysis of variance (ANOVA) was employed, using IG, time and their interaction. The assumptions of the applied statistical tests were ensured (ie, normality and homogeneity of variance for t-tests, ${ }^{73-75}$ normality and sphericity of variance for repeated measures ANOVA).$^{7677}$

Furthermore, analysis of covariance was conducted to assess the effect of the intervention on the outcomes after adjusting for confounding variables such as age and sex of the family caregivers, the presence of children and the availability of secondary caregivers.

Statistical significance was set at $\mathrm{p}<0.05$. The last observation carried forward method was used to compensate for missing data.

\section{RESULTS}

Between December 2019 and May 2020, 139 participants were evaluated to determine their eligibility for the study. Of them, 17 participants did not meet the inclusion criteria, eight refused to participate, and four stroke survivors died before randomisation. One hundred and ten family caregivers were allocated to either the IG $(n=55)$ or the CG $(\mathrm{n}=55)$. Of the 110 caregivers ( 50 in the IG and 47 in the CG), 97 (88.2\%) completed the study within 6 months. The intervention was completed at the end of November 2020, and the data of all 110 family caregivers were analysed; the reasons for dropout are displayed in figure 2. Six participants could not be contacted by the intervention nurses, and three participants did not attend the peer session. Moreover, four participants did not continue the study because their stroke survivors died.

\section{Characteristics of the study participants}

The baseline characteristics of both stroke survivors and their family caregivers were well balanced between the two groups, as shown in tables 1 and 2. The mean age of the stroke survivors was $65.66(\mathrm{SD} \pm 10.09)$ years, and the majority $(\mathrm{n}=86,78.2 \%)$ were men. For most participants 'current stroke' referred to the first occurrence of stroke, except for three in the IG. The proportion of stroke survivors with $\mathrm{mRS}$ scores of 4 and $5(\mathrm{n}=80,72.7 \%)$ was greater than that of stroke survivors with $\mathrm{mRS}$ scores of $3(n=30$, 


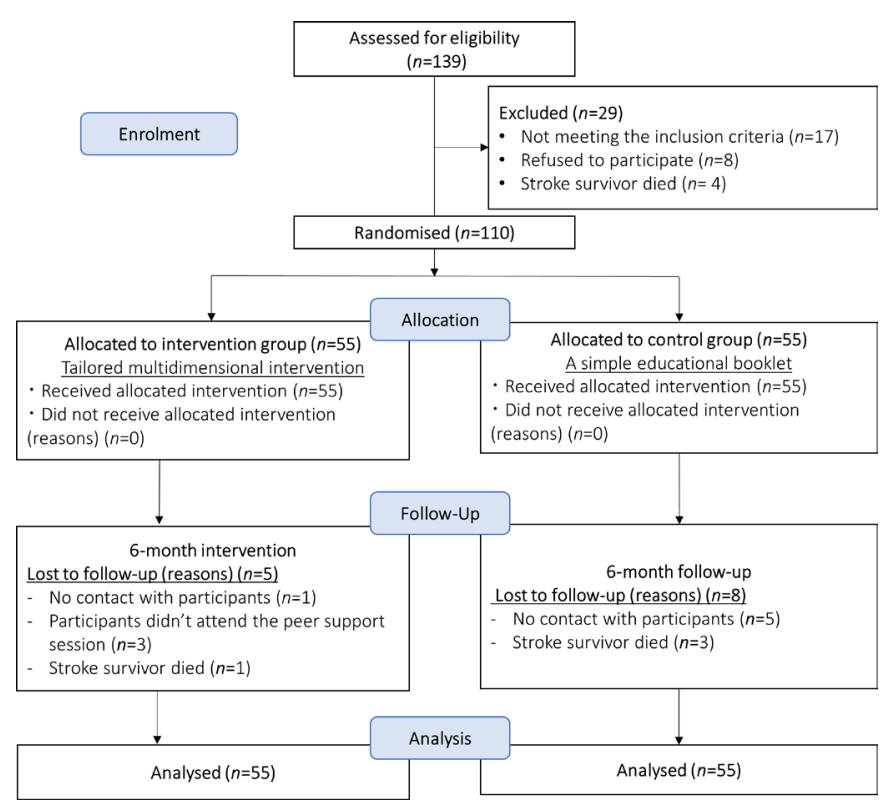

Figure 2 Diagram according to Consolidated Standards of Reporting Trials (CONSORT).

$27.3 \%)$. Majority of the patients had chronic comorbidities $(n=76,69.1 \%)$, while $11(10.0 \%)$ had cognitive impairment. All family caregivers who participated in this study had direct family kinship with the stroke survivors, and most were women $(n=82,74.5 \%)$, married $(n=98$, $89.1 \%)$, and lived in the same house as the stroke survivor $(\mathrm{n}=96,87.3 \%)$. Two-thirds of the caregivers had children $(\mathrm{n}=73,66.4 \%)$. The median number of caregiving hours provided by family caregivers was 56 (7-90) hours/week.

\section{Study outcomes between the two groups in different time points}

As indicated in table 3 , there were no statistically significant differences between the two groups in terms of care burden and QoL ( $\mathrm{p} \geq 0.05)$ at baseline (T0).

Regarding the care burden, initially, it slightly decreased from baseline (T0) to 3 months (T1) in the IG, while it slightly increased in the CG from baseline (T0) to follow-up (T2) figure 3. The t-tests showed no statistically significant differences in the care burden between the two groups at $\mathrm{T} 1$ and $\mathrm{T} 2(\mathrm{p} \geq 0.05)$. In terms of the effect of the intervention over time, no significant differences were observed in the interaction (group $\times$ time), within groups, or between groups, as shown in table 3 (all $\mathrm{p} \geq 0.05$ ).

Concerning QoL, figure 4 shows that both the physical and environmental domains scores declined over time in the IG and CG. On the contrary, the psychological and social relationship domain scores increased over time in the IG, whereas those in the CG decreased. T-tests revealed no significant differences between the two groups at T1 and T2 in the four domains of QoL (all $\mathrm{p} \geq 0.05$ ), as shown in table 3 . There were no significant differences within groups or between groups for all domains of QoL, as shown in table 3 (all $\mathrm{p} \geq 0.05$ ), while the effects of group and time interaction on both the psychological and social relationship domains were significant, $\mathrm{F}(1.37,147.73)=13.196, \mathrm{p}<0.001, \eta \mathrm{p}^{2}=0.109$ and $\mathrm{F}(1.35,145.32)=3.984, \mathrm{p}=0.036, \eta \mathrm{p}^{2}=0.036$, respectively.

After adjusting for confounding variables, analysis of covariance showed that the intervention did not have significant effects on care burden and QoL.

\section{DISCUSSION}

To date, this was the first RCT developed in Egypt that offered an intervention to family caregivers who provided care to stroke survivors at home. This intervention was premised on the recommendations highlighted in multiple systematic literature reviews and the proven advantages of previous interventions in community settings. ${ }^{1331}$ However, the study results show that the care burden for caregivers who received the intervention did not significantly differ from those allocated to the CG. Regarding the secondary outcome (QoL), the interaction (group $\times$ time) had a significant effect on psychological and social relationship domains. Nonetheless, no significant differences were observed between the groups at separate time points ( $\mathrm{T} 1$ and $\mathrm{T} 2$ ) or within the groups over time (T0-T2).

The findings of this study may be attributed to several factors. First, the lack of a structured healthcare system in Egypt, which does not support stroke survivors and their caregivers, is the most relevant factor that may have affected our study results. This interpretation is consistent with previous studies conducted in other countries that, like Egypt, lack a structured system for providing support/care for this target group. These studies also included interventions that adopted skill-building, psychoeducational or peer-support approaches. Consider the case of two RCTs conducted in Brazil $^{78}$ and Taiwan, ${ }^{79}$ wherein the first study adopted education, skill-building, and emotional approaches to support family caregivers in managing stroke survivors at home. While the second offered weekly personalised home-based interventions to guide caregivers in improving stroke survivors' body functions and level of independence. The results of both studies revealed that the care burden between the IG and CG was not significantly different, moreover, it increased in both IG and CG at the follow-up time points. On the contrary, interventions that produced significant effects on family caregivers and stroke survivors were conducted in countries with structured long-term care services. ${ }^{80}$ Similar to our study, a training programme for family caregivers of stroke survivors in Portugal was delivered by nurses and included a 3-month follow-up; the programme aimed at enabling the caregiver to adjust to the strokerelated health demands at home. Contrary to our study, the results showed a significant reduction in the care burden of caregivers. This result can be attributed to the fact that most of the study participants were either supported by another person in performing the care tasks or received assistance from an institution. ${ }^{81}$ 
Table 1 Baseline characteristics of stroke survivors in both the intervention and control groups

\begin{tabular}{|c|c|c|c|}
\hline Variables & Total & $\begin{array}{l}\text { Intervention group } \\
(n=55)\end{array}$ & $\begin{array}{l}\text { Control group } \\
(n=55)\end{array}$ \\
\hline Age (years old) ${ }^{*}$ & $65.66 \pm 10.09$ & $65.75 \pm 10.34$ & $65.58 \pm 9.94$ \\
\hline \multicolumn{4}{|l|}{ Gender (\%)† } \\
\hline Male & $86(78.2)$ & $45(81.8)$ & $41(74.5)$ \\
\hline Female & $24(21.8)$ & $10(18.2)$ & $14(25.5)$ \\
\hline \multicolumn{4}{|l|}{ Marital status (\%)† } \\
\hline Married & $49(44.5)$ & $22(40.0)$ & $27(49.1)$ \\
\hline Divorced & $3(2.7)$ & $1(1.8)$ & $2(3.6)$ \\
\hline Widowed & $58(52.7)$ & $32(58.2)$ & $26(47.3)$ \\
\hline \multicolumn{4}{|l|}{ Education level (\%)† } \\
\hline Cannot read and write & $13(11.8)$ & $9(16.4)$ & $4(7.3)$ \\
\hline Elementary & $40(36.4)$ & $21(38.2)$ & $19(34.5)$ \\
\hline Preparatory & $4(3.6)$ & $1(1.8)$ & $3(5.5)$ \\
\hline Secondary & $38(34.5)$ & $17(30.9)$ & 21 (38.2) \\
\hline Bachelor & $15(13.6)$ & $7(12.7)$ & $8(14.5)$ \\
\hline \multicolumn{4}{|l|}{ Residence (\%)† } \\
\hline Urban & $43(39.1)$ & $23(41.8)$ & $20(36.4)$ \\
\hline Rural & 67 (60.9) & 32 (58.2) & 35 (63.6) \\
\hline First-ever stroke (\%)† & 107 (97.3) & $52(94.5)$ & $55(100.0)$ \\
\hline \multicolumn{4}{|c|}{ Duration of the current stroke (months) $\dagger$} \\
\hline 1 & $28(25.5)$ & $15(27.3)$ & $13(23.6)$ \\
\hline 2 & $10(9.1)$ & $5(9.1)$ & $5(9.1)$ \\
\hline 3 & $14(12.7)$ & $6(10.9)$ & $8(14.5)$ \\
\hline 4 & $15(13.6)$ & $7(12.7)$ & $8(14.5)$ \\
\hline 5 & $29(26.4)$ & $13(23.6)$ & $16(29.1)$ \\
\hline 6 & $14(12.7)$ & $9(16.4)$ & $5(9.1)$ \\
\hline \multicolumn{4}{|l|}{ mRS score (\%)† } \\
\hline mRS: 3 & $30(27.3)$ & $15(27.3)$ & $15(27.3)$ \\
\hline mRS: 4 & $39(35.4)$ & $19(34.5)$ & $20(36.4)$ \\
\hline mRS: 5 & $41(37.3)$ & $21(38.2)$ & $20(36.4)$ \\
\hline $\begin{array}{l}\text { MMSE } \leq 20 \\
\text { (Cognitive impairment) (\%) } \dagger\end{array}$ & $11(10.0)$ & $6(10.9)$ & $5(9.1)$ \\
\hline Chronic diseases (\%)† & $76(69.1)$ & $40(72.7)$ & $36(65.5)$ \\
\hline \multicolumn{4}{|l|}{ Difficulties associated with stroke } \\
\hline Aphasia (\%)† & $45(40.9)$ & $26(47.3)$ & $19(34.5)$ \\
\hline Dysphagia (\%)† & $34(30.9)$ & $18(32.7)$ & $16(29.1)$ \\
\hline Hearing difficulty (\%)† & $44(40.0)$ & $18(32.7)$ & $26(47.3)$ \\
\hline Visual difficulty (\%)† & $58(52.7)$ & $26(47.3)$ & $32(58.2)$ \\
\hline
\end{tabular}

${ }^{*}$ Variables expressed as mean $\pm \mathrm{SD}$.

†Absolute number and percentage (\%).

MMSE, Mini-Mental State Examination; mRS, modified Rankin Scale.

Furthermore, the care burden of our study participants may have been influenced by the social determinants experienced by family caregivers in Egypt, such as poverty, unemployment and inadequate health services. ${ }^{48}$ The findings of our study indicate that the family caregivers' environmental QoL declined over time in both the groups. The environmental domain encompasses financial resources, home environment, physical safety and security, transportation, quality of health and social care, and opportunities for recreation and leisure 
Table 2 Baseline characteristics of family caregivers in both the intervention and control groups

\begin{tabular}{|c|c|c|c|}
\hline Variables & Total & $\begin{array}{l}\text { Intervention } \\
\text { group } \\
(n=55)\end{array}$ & $\begin{array}{l}\text { Control } \\
\text { group } \\
(n=55)\end{array}$ \\
\hline Age (years old) ${ }^{*}$ & $35(25-57)$ & $35(25-55)$ & $35(25-57)$ \\
\hline \multicolumn{4}{|l|}{ Gender (\%)† } \\
\hline Male & $28(25.5)$ & $13(23.6)$ & $15(27.3)$ \\
\hline Female & $82(74.5)$ & $42(76.4)$ & $40(72.7)$ \\
\hline \multicolumn{4}{|l|}{ Marital status (\%)† } \\
\hline Single & $5(4.5)$ & $3(5.5)$ & $2(3.6)$ \\
\hline Married & $98(89.1)$ & 48 (87.3) & $50(90.9)$ \\
\hline Divorced & $1(0.9)$ & $0(0)$ & $1(1.8)$ \\
\hline Widowed & $6(5.5)$ & $4(7.3)$ & $2(3.6)$ \\
\hline \multicolumn{4}{|l|}{ Education level (\%)† } \\
\hline Elementary & $11(10.0)$ & $4(7.3)$ & $7(12.7)$ \\
\hline Preparatory & 17 (15.5) & $10(18.2)$ & $7(12.7)$ \\
\hline Secondary & $51(46.4)$ & $26(47.3)$ & $25(45.5)$ \\
\hline Bachelor & $25(22.7)$ & $11(20.0)$ & $14(25.5)$ \\
\hline Postgraduate & $6(5.5)$ & $4(7.3)$ & $2(3.6)$ \\
\hline Employed (\%)† & $54(49.1)$ & $25(45.5)$ & $29(52.7)$ \\
\hline With children (\%)† & $73(66.4)$ & 39 (70.9) & $34(61.8)$ \\
\hline \multicolumn{4}{|l|}{ Income (\%)† } \\
\hline Sufficient & 34 (30.9) & $21(38.2)$ & $13(23.6)$ \\
\hline Insufficient & $76(69.1)$ & $34(61.8)$ & $42(76.4)$ \\
\hline Chronic diseases (\%)† & $13(11.8)$ & $5(9.1)$ & $8(14.5)$ \\
\hline \multicolumn{4}{|l|}{ Kinship to the stroke survivor (\%)† } \\
\hline Wife & $17(15.5)$ & $8(14.5)$ & $9(16.4)$ \\
\hline Son & $28(25.5)$ & $13(23.6)$ & $15(27.3)$ \\
\hline Daughter & $34(30.9)$ & $17(30.9)$ & $17(30.9)$ \\
\hline Daughter in law & $30(27.3)$ & $17(30.9)$ & $13(23.6)$ \\
\hline Sister & $1(0.9)$ & $0(0)$ & $1(1.8)$ \\
\hline Resides with the stroke survivor in the same house (\%)† & $96(87.3)$ & $46(83.6)$ & $50(90.9)$ \\
\hline Caregiving hours (per week)* & $56(7-90)$ & $56(7-84)$ & $60(10-90)$ \\
\hline Availability of secondary caregiver (\%)† & $95(86.4)$ & $48(87.3)$ & $47(85.5)$ \\
\hline
\end{tabular}

*Variables expressed as median (minimum-maximum).

†Variables expressed as absolute number and percentage (\%).

activities. Moreover, half of the study participants were unemployed. At the same time, more than two-thirds of the participants had low income, and most lived with stroke survivors (in the same house), were married and had social responsibilities. They had also only recently undertaken the caregiving responsibility and had apportioned a high number of hours to providing care. Additionally, all of the stroke survivors in our study had high levels of dependence, most were older adults, and more than two-thirds had other chronic illnesses-factors that also determine the care burden. ${ }^{26} 54{ }^{82-87}$ These findings reflect the social conditions of Egyptian family caregivers and the growing care demands of stroke survivors that are inherent to the concept of care burden and other negative stressors. ${ }^{32} 8889$

Another explanation for our findings is that, although our intervention was conducted sufficiently early (within 6 months of stroke occurrence $)^{5051}$ it was delivered in the community after hospital discharge. Whereas, studies indicate that interventions conducted before hospital discharge and continued post-discharge were more likely to achieve significant outcomes ${ }^{90}$ For instance, in an RCT conducted in Hong Kong to evaluate the effectiveness of an intervention programme on caregiving burden, which included nurse-led strength-oriented psychoeducation, similar to our study, the IG displayed a significantly lower 

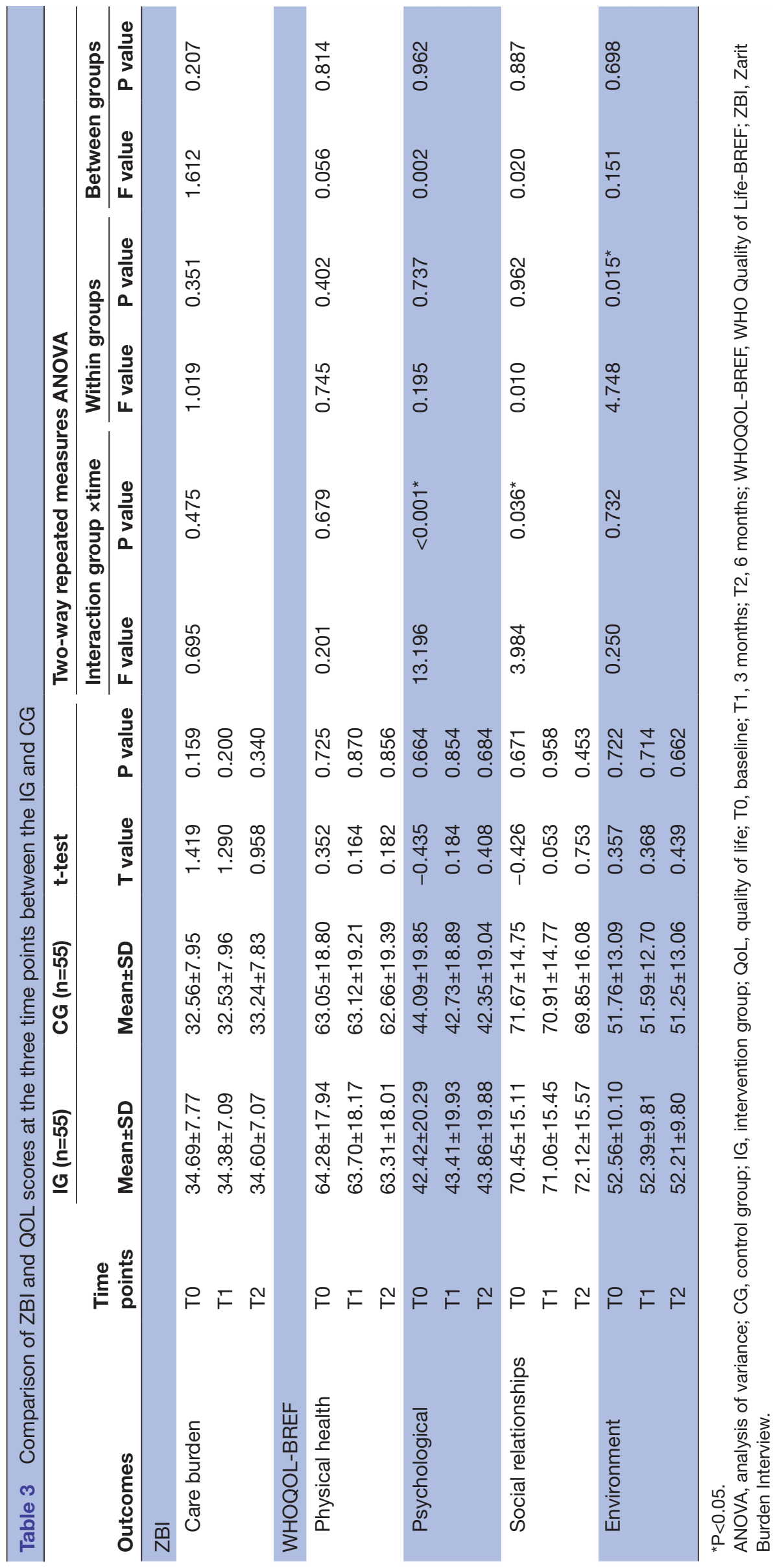


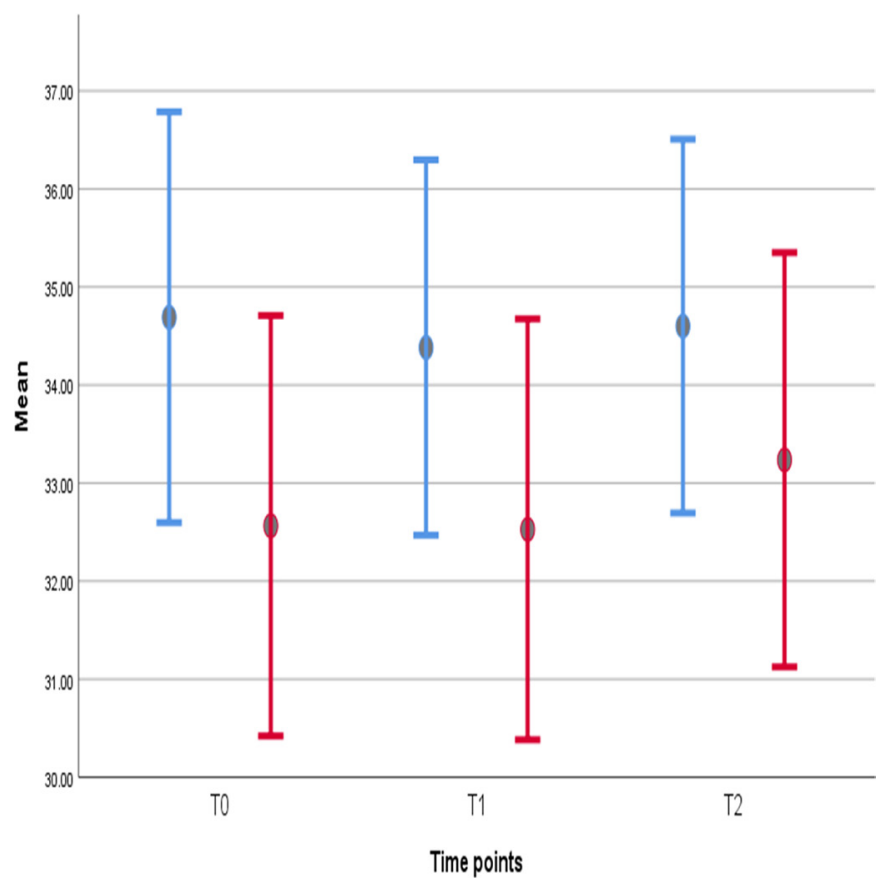

Figure 3 Mean of Zarit Burden Interview with SE bars ( $.5 \%$ $\mathrm{Cl}$ and $\pm 2 \mathrm{SE}$ ); T0, baselin ; T1, 3 months; T2, 6 months. $\uparrow$ Intervention group $(n=55) ;$ control group $(n=55)$.

level of care burden at 3-month postintervention. ${ }^{27}$ These significant results can be attributed to the two individual teaching sessions conducted at the hospital before the discharge of the survivor. Importantly, six additional intervention sessions continued at home immediately after hospital discharge.
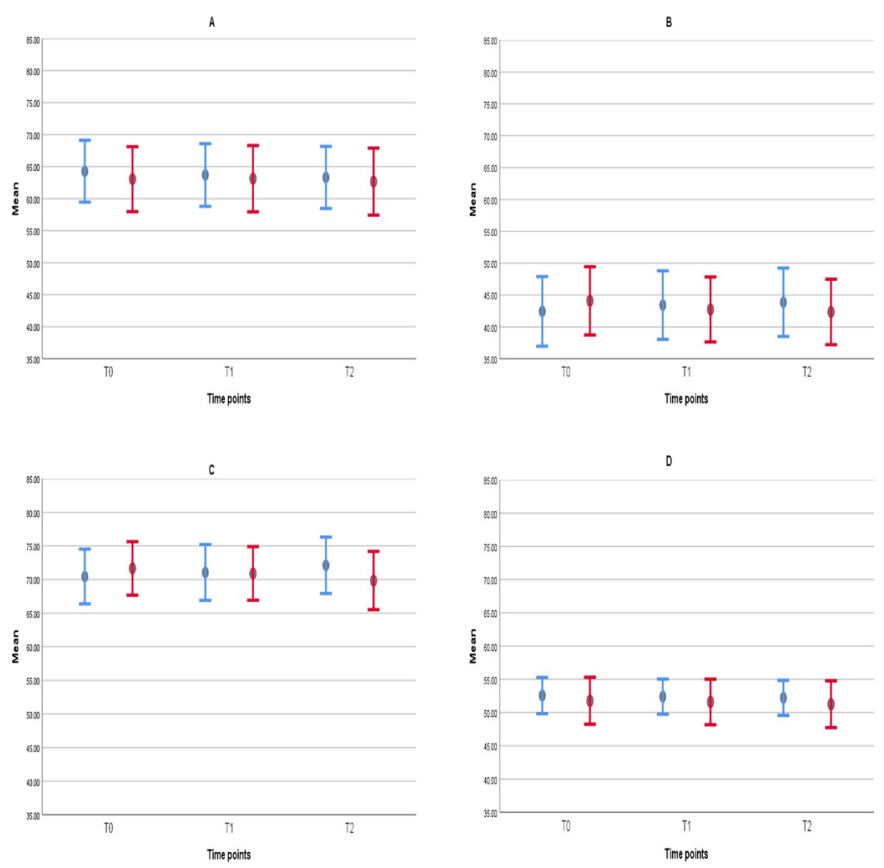

Figure 4 Means of four domains of WHO Quality of LifeBREF with SE bars (95\% Cl and \pm 2 SE). (A) Physical, (B) psychological, (C) social relationships, and (D) environment; T0, baseline; T1, 3 months; T2, 6 months.
Irrespective of our study results, which may be influenced by multiple factors (as discussed above), it is worth noting that the care burden in the IG decreased at T1 (3 months) compared with baseline. Additionally, both the psychological and social relationship domains of QoL in the IG improved over time compared with the CG. Group and time interaction also showed significant effects on both domains; these effects could be related to our intervention. The reported progress may be explained by the fact that our intervention included evidence-based components to some extent. Besides, the provision of 6 months of psychoeducation and skill-building also helps caregivers cope with their current situation.

Unfortunately, the care burden at T2 (6 months) returned to a similar level as the baseline, (T0) following a decrease after 3 months. Nevertheless, it was expected that it would decrease again as the intervention continued for another 3 months. This deterioration may have resulted from the varying intensity of the intervention throughout the study period: three home visits, three telephone calls and one peer support were delivered during the first 3 months, while only three telephone calls were provided in the next 3 months.

Finally, our intervention may not be adequate to meet the needs of caregivers, as they had anticipated. The intervention nurses often observed that some caregivers intended to show the nurses that they were truly suffering due to providing long-term care for stroke survivors at home and had expected to receive additional support beyond what was offered by the study intervention. Thus, some of the participants may not have completely engaged in the intervention in the ways we had expected.

\section{Limitations}

This study has several limitations that could highlight further interpretations of insignificant results. An important issue that should be emphasised is that the caregivers' rate of compliance with the intervention was not measured. Thus, the effectiveness of the intervention could not be explored extensively. Additionally, caregivers, patients and the public were not adequately involved in the development of the intervention. Moreover, the feasibility and acceptability of the intervention for the participants were not sufficiently assessed. The amount of intervention throughout the intervention period was also inconsistent. Furthermore, the unblinded outcome assessments and self-reported questionnaires used could also be possible sources of anticipatory bias.

Finally, one peer-support session may not be sufficient for the delivery of the intervention. Unfortunately, we could not hold more than one session because of the participants' limited time. The idea of peer support was not easily accepted by the participants, as caregivers did not participate actively in the peer-support discussion, and other caregivers did not even attend. This finding could be attributed to two major reasons. First, family caregivers preferred not to share their personal experiences and feelings with strangers. Second, it is difficult to build a strong relationship with peers in only 
one peer-support session. Moreover, the interventions were delivered by nurses with varying levels of enthusiasm; hence, some sessions may not have been delivered as effectively as desired.

Given the wider context of the setting during the study period, we must consider that the COVID-19 pandemic could have affected the study in the following manner:

- Researchers and intervention nurses applied standard precautions to prevent the spread of the virus. Yet, some caregivers did not attend the peer-support sessions, and other caregivers were afraid of the intervention nurses during their home visits.

- Two intervention nurses preferred not to be involved in the delivery of the intervention. They were replaced by four nurses who required additional preparation and training.

- The trial extended 3 months over the anticipated completion date.

\section{CONCLUSION}

In conclusion, we performed the first RCT directed at family caregivers of stroke survivors in Egypt at the community level. In response to the research question of our study, the results showed no significant differences between the IG and CG in terms of reducing the family caregivers' burden or improving their QoL. Family caregivers in Egypt may need more than psychoeducation, skill-building or peer-support interventions to reduce their care burden and improve other outcomes. In brief, although our findings are not generalisable, this type of intervention may not be sufficiently effective for family caregivers of stroke survivors in the Egyptian context.

\section{RECOMMENDATIONS}

We recommend further testing of various interventions in the Egyptian context. These interventions should be designed in accordance with evidence-based recommendations, as in our intervention, but should also address the limitations of our intervention. Besides, future initiatives should be established through the collaboration of multiple sectors to ensure adequate support. Moreover, we recommend including process evaluation during the implementation phase to ensure the comprehensiveness of interventions and to obtain an in-depth understanding of the findings. Finally, future studies should also use qualitative approaches to examine the meaning of 'care burden' and its determinants among the family caregivers of stroke survivors in the Egyptian context.

\footnotetext{
Author affiliations

${ }^{1}$ Graduate School of Biomedical and Health Sciences, Hiroshima University,

Hiroshima, Japan

${ }^{2}$ Community Health Nursing Department, Faculty of Nursing, Cairo University, Cairo, Egypt

${ }^{3}$ Psychiatric and Mental Health Nursing Department, Faculty of Nursing, Mansoura University, Mansoura, Egypt

${ }^{4}$ Nursing Department, College of Health and Sport Sciences, University of Bahrain, Manama, Bahrain
}

${ }^{5}$ New Mansoura General Hospital, Neurology, Egypt Ministry of Health and Population, Mansoura, Egypt

${ }^{6}$ Neurology, Faculty of Medicine, Mansoura University, Mansoura, Egypt ${ }^{7}$ Public Health, Faculty of Medicine, Mansoura University, Mansoura, Egypt

${ }^{8}$ Psychiatry, Faculty of Medicine, Mansoura University, Mansoura, Egypt

Acknowledgements The authors thank the Cultural Affairs and Missions Sector, Ministry of Higher Education, Cairo, Egypt, for providing the principal investigator with full scholarship (ID: KH-449) to study in Japan. The authors thank Hatano Rehabilitation Group, Aki-Ku, Hiroshima, Japan, for support and training in whole family assessments and care management processes. The authors would like to thank Editage for providing editorial assistance.

Contributors MAE is the principal investigator responsible for the initial draft of this manuscript and organising and implementing the study. MAE is responsible for the overall content as the guarantor. MAE, MM, MMR and MK formulated the study design, developed the intervention and defined the study outcomes. MA, MHK and $\mathrm{HZ}$ helped in recruitment and retention of the study participants. MAE, AHE-M, $M Z$, MK and A-HE-G calculated the sample size and decided on the data analysis plan. MAE, MA, MHK, AHE-M, MZ and $\mathrm{HZ}$ designed the tailored multidimensional plans and ensure the implementation and follow-up. MM, MMR and MK periodic review of the implementation process and give valuable feedback. MAE, MA, MHK, AHE-M and $M Z$ trained the intervention nurses. MAE, AHE-M, MZ and HZ were involved in the data collection. MAE, AHE-M, MZ, MM, MA and A-HE-G conducted critical revisions to data analysis. MAE wrote the discussion, and it was revised and modified by MM, MMR, MK and MA. All authors have read and approved the final manuscript.

Funding The authors have not declared a specific grant for this research from any funding agency in the public, commercial or not-for-profit sectors.

Competing interests None declared.

Patient consent for publication Consent obtained directly from patient(s)

Ethics approval Details regarding ethical issues are provided in the study protocol. This study was approved by the Research Ethics Committee of the Faculty of Nursing, Mansoura University, Mansoura, Egypt, in September 2019 (P.0195). Written informed consent was obtained from the stroke survivors and their family caregivers, which ensured voluntary and anonymous participation, as well as freedom to withdraw from the study at any time. Participants gave informed consent to participate in the study before taking part.

Provenance and peer review Not commissioned; externally peer reviewed.

Data availability statement Data are available in a public, open access repository. Dataset is available from the Dryad repository, D0l:[10.5061/dryad. gf1vhhmm5].https://doi.org/10.5061/dryad.gf1vhhmm5

Supplemental material This content has been supplied by the author(s). It has not been vetted by BMJ Publishing Group Limited (BMJ) and may not have been peer-reviewed. Any opinions or recommendations discussed are solely those of the author(s) and are not endorsed by BMJ. BMJ disclaims all liability and responsibility arising from any reliance placed on the content. Where the content includes any translated material, BMJ does not warrant the accuracy and reliability of the translations (including but not limited to local regulations, clinical guidelines, terminology, drug names and drug dosages), and is not responsible for any error and/or omissions arising from translation and adaptation or otherwise.

Open access This is an open access article distributed in accordance with the Creative Commons Attribution Non Commercial (CC BY-NC 4.0) license, which permits others to distribute, remix, adapt, build upon this work non-commercially, and license their derivative works on different terms, provided the original work is properly cited, appropriate credit is given, any changes made indicated, and the use is non-commercial. See: http://creativecommons.org/licenses/by-nc/4.0/.

\section{ORCID iDs}

Mahmoud Ahmed Elsheikh http://orcid.org/0000-0002-4256-9516

Md Moshiur Rahman http://orcid.org/0000-0002-5475-986X

Ahmed Hashem EL-Monshed http://orcid.org/0000-0002-0085-4685

\section{REFERENCES}

1 Benjamin EJ, Muntner P, Alonso A, et al. Heart disease and stroke Statistics -2019 update: a report from the American heart association. Circulation 2019;139:56-28. 
2 Andrew NE, Kilkenny MF, Naylor R, et al. The relationship between caregiver impacts and the unmet needs of survivors of stroke. Patient Prefer Adherence 2015;9:1065-73.

3 Camak DJ. Addressing the burden of stroke caregivers: a literature review. J Clin Nurs 2015;24:2376-82.

4 Gbiri CA, Olawale OA, Isaac SO. Stroke management: informal caregivers' burdens and strians of caring for stroke survivors. Ann Phys Rehabil Med 2015;58:98-103.

5 Adelman RD, Tmanova LL, Delgado D, et al. Caregiver burden: a clinical review. JAMA 2014:311:1052-60.

6 Kojima K, Nakayama T, Watanabe A. Activities of daily living and quality of life assessment during home-based rehabilitation - a multi-institutional study. Japanese J Comprehensive Rehabilitation 2017;8:30-6.

7 Jarvis A, Smith M, McAlpine L, et al. Caring for the carer of someone who has had a stroke: findings from an innovative project. Int $J$ Ther Rehabil 2019;26:1-11.

8 Gertrude N, Kawuma R, Nalukenge W, et al. Caring for a stroke patient: The burden and experiences of primary caregivers in Uganda - A qualitative study. Nurs Open 2019:6:1551-8.

9 Ellis G, Mant J, Langhorne P, et al. Stroke liaison workers for stroke patients and carers: an individual patient data meta-analysis. Cochrane Database Syst Rev 2010;5:CD005066.

10 Forster A, Brown L, Smith J, et al. Information provision for stroke patients and their caregivers. Cochrane Database Syst Rev 2012;11:CD001919.

11 Grant JS, Clay OJ, Keltner NL. Does caregiver well-being predict stroke survivor depressive. Top Stroke Rehabil 2013;20:1-13.

12 Elsheikh MA, Moriyama M, Rahman MM, et al. Effect of a tailored multidimensional intervention on the care burden among family caregivers of stroke survivors: study protocol for a randomised controlled trial. BMJ Open 2020;10:e041637.

13 Bakas T, Clark PC, Kelly-Hayes M, et al. Evidence for stroke family caregiver and dyad interventions. Stroke 2014;45:2836-52.

14 Bakas T, McCarthy M, Miller ET. Update on the state of the evidence for stroke family caregiver and dyad interventions. Stroke 2017;48:e122-5.

15 Mou H, Wong MS, Chien WT. Effectiveness of dyadic psychoeducational intervention for stroke survivors and family caregivers on functional and psychosocial health: a systematic review and meta-analysis. Int J Nurs Stud 2021:120:103969.

16 Frank A. The latest national clinical guideline for stroke. Clin Med 2017; $17: 478$

17 Forster A, Young J, Nixon J, et al. A cluster randomized controlled trial of a structured training programme for caregivers of inpatients after stroke (TRACS). Int J Stroke 2012;7:94-9.

18 White CL, Cantu AG, Trevino MM. Interventions for caregivers of stroke survivors: an update of the evidence. Clin Nurs Stud 2015;3:87-95.

19 Tsai P-C, Yip P-K, Tai JJ, et al. Needs of family caregivers of stroke patients: a longitudinal study of caregivers' perspectives. Patient Prefer Adherence 2015;9:449-57.

$20 \mathrm{Li}$ X, Xia X, Wang P, et al. Needs and rights awareness of stroke survivors and caregivers: a cross-sectional, single-centre questionnaire survey. BMJ Open 2017;7:e013210

21 Denham AMJ, Wynne O, Baker AL, et al. An online survey of informal caregivers' unmet needs and associated factors. PLoS One 2020;15:e0243502

22 Grant JS, Hunt CW, Steadman L. Common caregiver issues and nursing interventions after a stroke. Stroke 2014;45:151-3.

23 Panzeri A, Rossi Ferrario S, Vidotto G. Interventions for psychological health of stroke caregivers: a systematic review. Front Psychol 2019;10:10.

24 Chuluunbaatar E, Pu C, Chou Y-J. Changes in caregiver burden among informal caregivers of stroke patients in Mongolia. Top Stroke Rehabil 2017;24:314-21.

25 Tosun ZK, Temel M. Burden of caregiving for stroke patients and the role of social support among family members: an assessment through home visits. Int J Caring 2017;10:1696-704 http://www. internationaljournalofcaringsciences.org/docs/65_8 tosun $10 \_3$. pdf

26 Long NX, Pinyopasakul W, Pongthavornkamol K, et al. Factors predicting the health status of caregivers of stroke survivors: a crosssectional study. Nurs Health Sci 2019;21:262-8.

27 Cheng HY, Chair SY, Chau JPC. Effectiveness of a strength-oriented psychoeducation on caregiving competence, problem-solving abilities, psychosocial outcomes and physical health among family caregiver of stroke survivors: a randomised controlled trial. Int $J$ Nurs Stud 2018;87:84-93.

28 Miller EL, Murray L, Richards L, et al. Comprehensive overview of nursing and interdisciplinary rehabilitation care of the stroke patient: a scientific statement from the American heart association. Stroke 2010;41:2402-48.

29 Morris R, Morris P. Participants' experiences of hospital-based peer support groups for stroke patients and carers. Disabil Rehabil 2012;34:347-54.

30 Roy C, Whetsell MV, Frederickson K. The Roy adaptation model and research. Nurs Sci Q 2009;22:209-11.

31 Cheng HY, Chair SY, Chau JP-C. The effectiveness of psychosocial interventions for stroke family caregivers and stroke survivors: a systematic review and meta-analysis. Patient Educ Couns 2014;95:30-44.

32 Hekmatpou D, Baghban EM, Dehkordi LM. The effect of patient care education on burden of care and the quality of life of caregivers of stroke patients. J Multidiscip Healthc 2019;20:211-7.

33 Clark NM, Rakowski W. Family caregivers of older adults: improving helping skills. Gerontologist 1983;23:637-42.

34 Gitlin LN, Corcoran M, Winter L, et al. A randomized, controlled trial of a home environmental intervention: effect on efficacy and upset in caregivers and on daily function of persons with dementia. Gerontologist 2001;41:4-14.

35 Cameron JI, Naglie G, Silver FL, et al. Stroke family caregivers support needs change across the care continuum: a qualitative study using the timing it right framework. Disabil Rehabil 2013;35:315-24.

36 Greenwood N, Mackenzie A, Harris R, et al. Perceptions of the role of general practice and practical support measures for carers of stroke survivors: a qualitative study. BMC Fam Pract 2011;12:57.

37 Grawburg M, Howe T, Worrall L, et al. A qualitative investigation into third-party functioning and third-party disability in aphasia: positive and negative experiences of family members of people with aphasia. Aphasiology 2013;27:828-48

38 Pitthayapong S, Thiangtam W, Powwattana A, et al. A community based program for family caregivers for post stroke survivors in Thailand. Asian Nurs Res 2017;11:150-7.

39 Kniepmann K, Cupler MH. Occupational changes in caregivers for spouses with stroke and aphasia. British Journal of Occupational Therapy 2014;77:10-18

40 Winkler M, Bedford V, Northcott S, et al. Aphasia blog talk: how does stroke and aphasia affect the carer and their relationship with the person with aphasia? Aphasiology 2014;28:1301-19.

41 Jones L, Morris R. Experiences of adult stroke survivors and their parent carers: a qualitative study. Clin Rehabil 2013;27:272-80.

42 Green TL, King KM. Experiences of male patients and wifecaregivers in the first year post-discharge following minor stroke: a descriptive qualitative study. Int J Nurs Stud 2009;46:1194-200.

43 Quinn K, Murray CD, Malone C. The experience of couples when one partner has a stroke at a young age: an interpretative phenomenological analysis. Disabil Rehabil 2014;36:1670-8.

44 Festinger L. A theory of social comparison processes. Human Relations 1954;7:117-40.

45 Christensen ER, Golden SL, Gesell SB. Perceived benefits of peer support groups for stroke survivors and caregivers in rural North Carolina. N C Med J 2019;80:143-8.

46 Muller M, Toth-Cohen S, Mulcahey MJ. Development and evaluation of a hospital-based peer support group for younger individuals with stroke. Occup Ther Health Care 2014;28:277-95.

47 Grant S, Mayo-Wilson E, Montgomery P, et al. CONSORT-SPI 2018 explanation and elaboration: guidance for reporting social and psychological intervention trials. Trials 2018;19:1-18.

48 Capmas, 2020. Available: https://www.capmas.gov.eg/Pages/ Publications.aspx?page_id=5109\&Year=23354 [Accessed 28 May 2020].

49 Chan A-W, Tetzlaff JM, Altman DG, et al. Spirit 2013 statement: defining standard protocol items for clinical trials. Ann Intern Med 2013;158:200.

50 Jaracz K, Grabowska-Fudala B, Górna K, et al. Burden in caregivers of long-term stroke survivors: prevalence and determinants at 6 months and 5 years after stroke. Patient Educ Couns 2015;98:1011-6.

51 Han Y, Liu Y, Zhang X, et al. Chinese family caregivers of stroke survivors: determinants of caregiving burden within the first six months. J Clin Nurs 2017;26:4558-66.

52 Broderick JP, Adeoye O, Elm J. Evolution of the modified Rankin scale and its use in future stroke trials. Stroke 2017;48:2007-12.

53 Awad MM, El Gammal HA, Fahmy MT. Determinants of disabled elderly caregivers burden in Ismailia, Egypt. Med J Cairo Univ 2010;78:31-7 https://www.academia.edu/33308030/Determinants_ of Disabled Elderly Caregivers Burden in Ismailia_Egypt

54 Watanabe O, Chompikul J, Kawamori M. Predictors of family caregiver burden in caring for older people in the urban district of Nakhon Ratchasima Province, Thailand. J Int Health 2019;34:217-28. 
55 Ferreira da Costa T, de Freitas Macêdo Costa KN. Burden over family caregivers of elderly people with stroke. Rev Esc Enferm USP 2015;19:350-5.

56 Kumar R, Burden AN. Its predictors and quality of life in caregivers of stroke survivors at rural community, Punjab, India. Journal of Neurology and Neurorehabilitation Research 2016;1:1-7.

57 Craig P, Dieppe P, Macintyre S, et al. Developing and evaluating complex interventions: the new medical Research Council guidance. Int J Nurs Stud 2013;50:587-92.

58 Moore GF, Audrey S, Barker M, et al. Process evaluation of complex interventions: medical Research Council guidance. BMJ 2015;350:h1258.

59 Hoffmann TC, Glasziou PP, Boutron I, et al. Better reporting of interventions: template for intervention description and replication (TIDieR) checklist and guide. BMJ 2014;348:g1687.

60 Bédard M, Molloy DW, Squire L, et al. The Zarit burden interview: a new short version and screening version. Gerontologist 2001;41:652-7.

61 World Health Organisation. Programme on mental health: WHOQOL user manual. Geneva, Switzerland: WHO, 1998.

62 Caro CC, Costa JD, Da Cruz DMC. Burden and quality of life of family caregivers of stroke patients. Occup Ther Health Care 2018;32:154-71.

63 Hu P, Yang Q, Kong L, et al. Relationship between the anxiety/ depression and care burden of the major caregiver of stroke patients. Medicine 2018:97:e12638.

64 Oni OD, Olagunju AT, Okpataku Cl, et al. Predictors of caregiver burden after stroke in Nigeria: effect on psychosocial well-being Indian J Psychiatry 2019;61:457-64.

65 Bachner YG. Preliminary assessment of the psychometric properties of the abridged Arabic version of the Zarit burden interview among caregivers of cancer patients. Eur J Oncol Nurs 2013;17:657-60.

66 Rawat M, Sharma R, Goel D. Burden of stroke survivors on caregiver and quality of life. Int J Curr Res 2017;9:60683-6 https://www. researchgate.net/publication/321569436_BURDEN_OF_STROKE_ SURVIVORS ON CAREGIVER AND QUALITY OF LIFE

67 Dalky HF, Meininger JC, Al-Ali NM. The reliability and validity of the Arabic World Health organization quality of life-BREF instrument among family caregivers of relatives with psychiatric illnesses in Jordan. J Nurs Res 2017;25:224-30.

68 Faul F, Erdfelder E, Lang A-G, et al. G*Power 3: a flexible statistical power analysis program for the social, behavioral, and biomedical sciences. In: Behavior research methods. Madison (WI: Psychonomic Society Inc, 2007: 39. 175-91.

69 Karagiozi K, Papaliagkas V, Giaglis G, et al. Combined intervention for caregivers of patients with dementia: a randomized controlled trial. IJARP 2014;1:77-95.

70 Schure LM, van den Heuvel ETP, Stewart RE, et al. Beyond stroke: description and evaluation of an effective intervention to support family caregivers of stroke patients. Patient Educ Couns 2006;62:46-55

71 Krysik J, Finn J. Research for effective social work practice. Abingdon: Routledge, 2013.

72 Detry MA, Lewis RJ. The intention-to-treat principle how to assess the true effect of choosing a medical treatment. JAMA 2020;312:85-6.

73 Doane DP, Seward LE. Measuring Skewness: a forgotten statistic? J Stat Educ 2011:19:1-18.
74 Cramer D. Fundamental statistics for social research: step-by-step calculations and computer techniques using SPSS for windows. 1 edn. Routledge, 1997.

75 Howitt D, Cramer D. First steps in research and statistics: a practical workbook for psychology students. 1 edn. Routledge, 2000.

76 Shapiro SS, Wilk MB. An analysis of variance test for normality (complete samples). Biometrika 1965;52:591-611.

77 Razali NM, Wah YB. Power comparisons of Shapiro-Wilk, Kolmogorov-Smirnov, Lilliefors and Anderson-Darling tests. J Stat Model Anal 2011;2:21-33 https://scholar.google.com/scholar lookup?journal=J+Stat+Model+Anal\&title=Power+comparisons+ of+Shapiro-Wilk,+Kolmogorov-Smirnov,+Lilliefors+and+AnsersonDarling+tests\&author $=\mathrm{NM}+$ Razali\&author $=\mathrm{YB}+\mathrm{Wah} \&$ volume $=22$ \& publication year $=2011$ \&pages $=21-33 \&$

78 Day CB, Bierhals CCBK, Mocellin D, et al. Nursing Home Care Intervention Post Stroke (SHARE) 1 year effect on the burden of family caregivers for older adults in Brazil: A randomized controlled trial. Health Soc Care Community 2021;29:56-65.

79 Wang T-C, Tsai AC, Wang J-Y, et al. Caregiver-mediated intervention can improve physical functional recovery of patients with chronic stroke: a randomized controlled trial. Neurorehabil Neural Repair 2015;29:3-12.

80 Fu R, Noguchi H, Kawamura A, et al. Spillover effect of Japanese long-term care insurance as an employment promotion policy for family caregivers. J Health Econ 2017;56:103-12.

81 Araújo O, Lage I, Cabrita J, et al. Training informal caregivers to care for older people after stroke: a quasi-experimental study. J Adv Nurs 2018;74:2196-206.

82 Boonsin S, Deenan A, Wacharasin C. Factors influencing the burden of family caregiving for survivors of stroke. Pacific Rim Int J Nurs Res 2020;25:102-13 https://nurse.buu.ac.th/rsh/file/journal/0000001147. pdf

83 Nunes DP, de Brito TRP, Duarte YA de O. Caregivers of elderly and excessive tension associated with care: evidence from the Sabe study. Rev Bras Epidemiol 2018;21:e180020.

84 Badaru UM, Ogwumike OO, Adeniyi AF, et al. Determinants of caregiving burden and quality of life of informal caregivers of African stroke survivors: literature review. Int J Disabil Hum Dev 2017; 16:249-58.

85 Celik A, Kara B, AIMS. The relationship between the stroke survivors functional status and their informal caregivers' burden and quality of life. AIMS Med Sci 2019;6:115-27.

86 Kaur P, Bhalla A, Katyal P, et al. Strain among the family caregivers of patients with stroke. J Perioper Crit Intensiv Care Nurs 2018;04:2-5.

87 Oliva-Moreno J, Peña-Longobardo LM, Mar J, et al. Determinants of informal care, burden, and risk of burnout in caregivers of stroke survivors: the CONOCES study. Stroke 2018;49:140-6.

88 Mourad GM, Zaki RA, Ali RA. Improving coping abilities among caregivers of patients with cerebrovascular stroke. J Educ Pract 2014;5:8-20

89 Ghazawy ER, Mohammed ES, Mahfouz EM, et al. Determinants of caregiver burden of persons with disabilities in a rural district in Egypt. BMC Public Health 2020;20:1156.

90 van den Berg M, Crotty M, Liu E, et al. Early supported discharge by caregiver-mediated exercises and e-health support after stroke: a proof-of-concept trial. Stroke 2016;47:1885-92. 